\title{
Maximum Radiated Emissions of Printed Circuit Board Using Analytical Methods
}

\author{
Mohd Zarar Mohd Jenu, Ahmed M. Sayegh, Syarfa Zahirah Sapuan \\ Research Center for Applied Electromagnetics, Universiti Tun Hussein Onn Malaysia, Malaysia
}

\begin{tabular}{l}
\hline \hline Article Info \\
\hline Article history: \\
Received Jun 12, 2017 \\
Revised Aug 20, 2017 \\
Accepted Oct 26, 2017 \\
\hline
\end{tabular}

Keyword:

Common mode

Diffrential mode

Imbalance difference model

Printed circuit board

Radiated emission

\begin{abstract}
The rapid progress of technology has imposed significant challenges on Printed Circuit Boards (PCB) designers. Once of those challenges is to satisfy the electromagnetic compatibility (EMC) compliance requirements. For that reason, EMC compliance must be considered earlier at the design stage for time and cost savings. Conventionally, full wave simulation is employed to check whether the designed PCB meets EMC standards or not. However, this method is not a suitable option since it requires intensive computational time and thus increasing the unit cost. This paper describes novel analytical models for estimating the radiated emissions (RE) of PCB. These models can be used to help the circuit designer to modify their circuit based on the maximum allowable RE comparing to the relevant EMC-RE standard limit. Although there are many RE sources on PCB, this paper focuses on the significant source of RE on PCB; namely PCB-traces. The trace geometry, termination impedance, dielectric type, etc. can be specified based on the maximum allowable emissions. The proposed models were verified by comparing the results of the proposed models with both simulation and experimental results. Good agreements were obtained between the analytically computed results and simulation/measurement results with accuracy of $\pm 3 \mathrm{~dB}$.
\end{abstract}

Copyright $@ 2017$ Institute of Advanced Engineering and Science. All rights reserved.

\section{Corresponding Author:}

Ahmed M. Sayegh,

Research Center for Applied Electromagnetics,

Universiti Tun Hussein Onn Malaysia (UTHM),

Parit Raja, Batu Pahat, 86400, Malaysia.

Email: ahmed_su2008@yahoo.com

\section{INTRODUCTION}

The design of electromagnetically compatible high speed electronic systems is one of the most significant challenges in the circuit industry. These systems normally comprises of one or more of Printed Circuit Board (PCB) which represent the heart of the electronic devices. The PCB can produce a huge amount of radiated emissions (RE) which may interfere with the operation of the nearby devices. These emissions exist due to differential-mode (DM) and common-mode (CM) currents. Thus, it is essential to take into account both the DM and CM RE.

The maximum RE can be quantified using several methods [1], [2]. One of those methods is to send the electronic device for measurement in a semi anechoic chamber (SAC). However, this method is expensive, rather iterative and time consuming task since it requires building the prototype first before performing the measurement process.

The other option is to use 3D numerical solver simulation. However, this approach also requires extensive computational resources to analyze the details of each trace structure. Furthermore, it provides relatively little physical insight of the RE mechanisms. Alternatively, simple novel analytical models are proposed in this paper for computing the maximum RE of that PCB-traces which can be used to as 
benchmark for the design process to find the optimum design that satisfy EMC requirements as described in the next sections.

\section{ESTIMATION OF RE FROM PCB-TRACES USING NOVEL ANALYTICAL MODELS}

The rapid increasing of the operating frequency of PCB to several gigahertz has enhanced PCBtraces to be efficient radiators of RE. Therefore, it is critically important to confirm that the produced REs do not exceed the RE standard limit [1]. The emission of the electrically short traces (trace length is less than one tenth wavelength) is computed based on the concept of Hertzian dipole antenna [2]. It is assumed that the DM current is uniformly distributed along the trace and thus it can be modeled as Hertzian dipole antenna. Now, this situation has changed and it is invalid for traces longer than one tenth wavelength. In this regard, the trace can be modeled as an array of Hertzian dipoles or as a travelling wave antenna as described in details in the next sections.

\subsection{DM-RE of Electrically Short PCB-traces}

On PCB, there are several sources of RE such as traces, electronic components, heatsink, etc. However, the PCB-traces are the dominant source of RE. These RE of PCB-traces are due to both DM and CM currents. Conventionally, the signal and return traces on PCB are modeled as two parallel Hertzian dipoles with opposite directions. The maximum DM electric field, $\left|\hat{E}_{D}\right|_{\max }$ is then given as [2]

$$
\left|\hat{E}_{D}\right|_{\max }=\frac{1.316 \times 10^{-14}\left|\hat{I}_{D}\right| f^{2} l s}{r}
$$

where $\left|\hat{I}_{D}\right|$ denotes DM current magnitude, $f$ is frequency, $l$ is the length of signal trace, $s$ is the distance between signal trace and return trace, and $r$ is the distance from the Device Under Test (DUT) to the observation point. Although CM current is much smaller than DM current, CM RE are the main contributor to the total RE in comparisons to DM RE [2]. For this reason, less attention has been given to DM emissions in the past. Today, the DM emissions are a significant contributor since PCBs currently operates in several gigahertz. The RE of PCB-traces is characterized based on the electrical length of the trace.

\subsection{DM-RE of Relatively Electrically Short PCB-traces $(\lambda / 10 \leq l \leq \lambda)$}

Conventionally, the REs of PCB traces are computed based on the Hertzian dipole antenna [2]. However, this method is limited to electrically short traces where the trace length is less than one tenth wavelength. Therefore, a method is proposed to overcome the deficiencies of conventional method by dividing the PCB trace into multiple segments. Each segment is modelled as a Hertzian dipole and then an array of Hertzian dipoles is obtained as shown in Figure 1.

The DM current distribution on electrically long PCB traces varies with frequency. In this situation, the phase delay in PCB traces should not be disregarded. Therefore, a PCB trace cannot be modelled as a single Hertzian dipole antenna; it should be modelled as an array of Hertzian dipole antenna.

The computation of total DM RE of PCB requires division of the trace into multiple electrically short segments, each with a length $\Delta \mathrm{x}$ according to wavelength of the maximum frequency of interest. The $\mathrm{RE}$ of each segment will then be estimated using Equation (1). Once DM current is known for each segment, the total RE can easily be computed by superimposing the RE generated by each segment. So, it is necessary to compute the DM current for the estimation of DM-RE.

The current distribution along the electrically long trace is not uniform. Thus, it can be chunked into many electrically small segments $(l<<\lambda)$. As the number of segments increases, the accuracy of calculation increases. However, it is not good idea since the computational time increases intensively as the number of segments increase. Therefore, it is critically important to determine the number of segments to achieve compromise between the accuracy and the required computational time.

The trace length must be divided into many equally small segments ( $\mathrm{N}$ segments). Each segment has length, $\Delta \mathrm{x}$, where $\Delta x=\lambda / 10$. Thus, the number of segments is computed as

$$
N=10 l / \lambda
$$


Once the DM current is calculated, Equation (1) can be applied to get the RE for all the frequencies considering the current change along the trace.

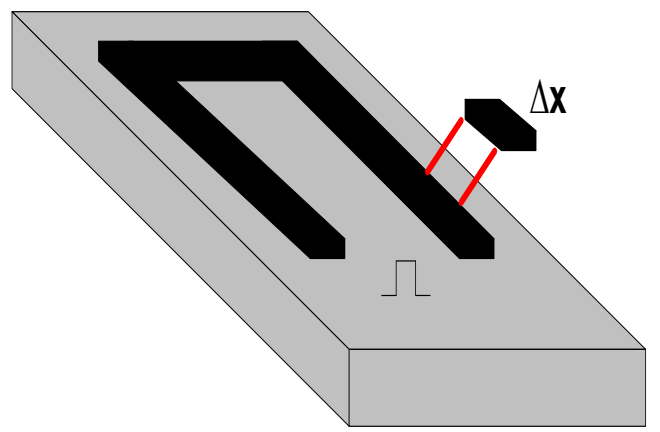

(a)

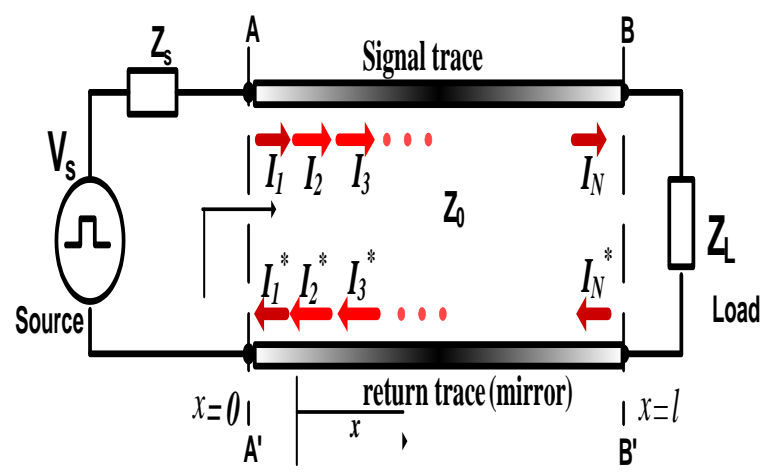

(b)

Figure 1. (a) Simple single-sided PCB for illustrating trace segmentation method, (b) equivalent circuit of the PCB under study

The currents $I_{1}, I_{2}, I_{3}, \ldots, I_{N}$ defines the DM currents of the segments along the signal trace whereas the $I_{1} *, I_{2} *, I_{3} *, \ldots, I_{N} *$ represents the current of the segment along the mirror trace. According to the expressions for the far-field of the Hertzian dipole, we can compute the radiated fields, $\vec{E}_{\text {total }}$, from all the segments in Figure 1 using the following expression

$$
\vec{E}_{\text {total }}(f)=\sum_{k=1}^{N} \vec{E}_{\text {signal }}(k, f)-\sum_{k=1}^{N} \vec{E}_{\text {mirror }}(k, f)
$$

where $\vec{E}_{\text {Signal }}$, and $\vec{E}_{\text {mirror }}$ are the radiated electric fields from the signal trace segments and mirror trace segments, respectively.

This method has been validated using several configurations of PCBs. The results of this proposed method are compared with full wave 3D simulation results which are used as benchmarks and obtained by commercial software Ansys HFSS as illustrated later in the results and discussion section.

Although this proposed method provides accurate results for estimating the DM RE from electrically long traces, it consumes relatively long computational time since it needs to calculate iteratively the RE for all the segments. This process is similar to the numerical calculation. Thus, it is considered an intermediate method for computing the RE. As a solution, a closed-form formulation was derived for estimating the DM from those traces [3]

$$
|\hat{E}|_{\max }=\frac{2.52 \times 10^{-6}\left|\hat{I}_{D}\right| f s}{r}[1-\cos (\pi l / \lambda)]
$$

where $l$ is the trace length and $\lambda$ is the wavelength of the signal of interest.

\subsection{Estimation of DM-RE from Electrically Long PCB-traces $(\boldsymbol{l}>\boldsymbol{\lambda})$}

In this method, the DM RE of electrically long traces are calculated based on the DM current flowing on the signal trace in conjunction of the far-field magnetic vector potential concept. In fact, the traces act as transmission line (TL). The DM current is then calculated using the TL theory as long as the operating frequency is within the QTEM range [2]. In contrast to previous studies [4], [5], the DM-RE can be computed directly by analytically determining the angle with maximum RE. The angle with maximum RE can be determined if the trace length and the wavelength of interest are known which further reduces the computational time significantly as shown in Figure 2.

The maximum DM RE of electrically long microstrip PCB-trace is computed as [6] 


$$
E_{\theta}=-j \omega \mu \frac{e^{-j k r}}{4 \pi r} \frac{V_{S}}{Z_{S}+Z_{0}} \frac{1}{1-\rho_{S} \rho_{L} e^{-j \beta 2 l}}\left[\left(\frac{1-e^{-j\left(\beta-k_{z}\right) l}}{\beta-k_{z}}+\rho_{L} e^{-j \beta 2 l} \frac{1-e^{j\left(\beta+k_{z}\right) l}}{\beta+k_{z}}\right)\right] \times F
$$

where $k_{z}=k_{0} \cos \theta, k_{0}$ is the wavenumber, $\mu$ is the permeability, $I(z)$ is the DM current a long z-direction and $r$ is the distance from PCB to the observation point. $\rho_{S}, \rho_{L}$ is the source-and load-refection coefficients respectively.

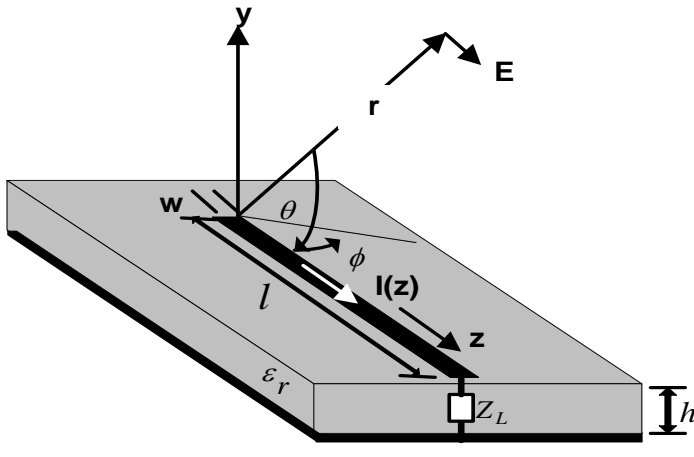

(a)

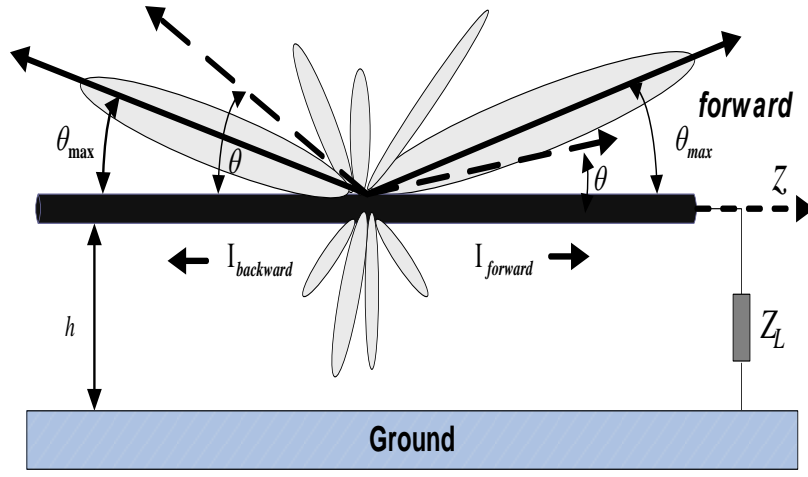

(b)

Figure 2. (a) Simple microstrip PCB for maximum DM-RE, (b) PCB-trace above ground plane equivalent to TWA [6]

The factor, F, can be written as [6]

$$
F=k_{0} h\left[\sin ^{2}(\theta) a_{\theta}-\cos \theta \sin \theta a_{r}\right]
$$

The angle that gives the maximum DM RE of electrically long PCB trace is given as [7]

$$
\theta_{\max }=\cos ^{-1}\left(1-\frac{\lambda}{2 l}\right)
$$

Several configurations of PCBs are employed to verify this derived expression as described in result and discussion section.

\section{CM RE OF PCB-TRACES USING DIPOLE ANTENNA THEORY}

Basically, the CM sources on PCB structure is due to the imbalance of the PCB-structure. Although $\mathrm{CM}$ currents is much smaller than DM current, it is the significant contributor to the overall emissions. This is due to the longer path that $\mathrm{CM}$ current flows through the circuit and its environment. The maximum CM $\mathrm{RE}, \vec{E}_{C, \max }$ of electrically short traces is estimated as [2]

$$
\vec{E}_{C, \max } \cong 1.257 \times 10^{-6} \frac{\hat{I}_{C} f l}{r}
$$

where $\hat{I}_{C}$ denotes the CM current. Although Equation (8) provides good estimate for CM RE, it is inaccurate for estimating the CM RE from electrically long PCB-traces. Thus, the authors had derived a closed form expression for computing the maximum CM RE of electrically long PCB-traces shorter than one wavelength as [8]

$$
\vec{E}_{C, \max } \cong \frac{120\left|\hat{I}_{C}\right|}{r}\left[1-\cos \left(\frac{\pi l}{\lambda_{0}}\right)\right]
$$


The expression (9) has overcome the limitation of (8). However, it is also limited to traces of maximum one wavelength. Therefore, a novel approach is presented for estimating CM RE from PCB-traces by combining the imbalance difference model and the asymmetrical dipole antenna as described in the next section.

In this research, the imbalance difference model [9] is adopted to locate and quantify the CM voltage source on PCB with electrically long trace while the signal trace and the ground plane in microstrip PCB are modelled as asymmetrical dipole antenna.

The imbalance difference factor $h$ can be defined as the ratio between the CM current on the signal trace to the total CM current. It can be expressed as [10]

$$
h=\frac{C_{\text {trace }}}{C_{\text {trace }}+C_{\text {board }}}
$$

where $C_{\text {trace }}, C_{\text {board }}$ are the stray capacitance of PCB-trace and PCB board respectively. The stray capacitance of the trace and ground plane can be computed either using closed-form expressions [91] or by using an electromagnetic simulation such as Ansys Q3D extractor. Once the stray capacitances are known, the $\mathrm{CM}$ voltage at the point where the imbalance changes can be computed as [10]

$$
V_{C M}(x)=\Delta h \times V_{D M}(x)
$$

where $\Delta h$ is the changed in the imbalance factor, $V_{D M}(x)$ is the DM voltage at the point where the change in imbalance occurs. So the CM voltage at the source/trace junction is computed as

$$
V_{C M}(0)=h_{2} V_{D M}(0)=\frac{C_{\text {trace }}}{C_{\text {trace }}+C_{\text {board }}} V_{D M}(0)
$$

In this paper, a simple microstrip PCB is employed to model CM of PCB-traces. This proposed PCB consist of one trace centred on PCB. It has source voltage and terminated with load impedance as shown in Figure 3. The trace and its ground plane are modelled as asymmetrical dipole antenna with parasitic CM voltage computed as Equation (12).

Two steps are required to quantify the CM RE of PCB-traces. The first step is to identify and quantify the CM voltage sources using IDM while the second step is to evaluate the CM RE from the equivalent structure using asymmetrical dipole antenna. In this paper, the CM current distribution is estimated according to functions introduced by R. King [11]. Referring to Figure 3, the asymmetrical antenna can be virtually decomposed into two symmetrical dipole antennas. Therefore, each branch can be analysed independently since the geometrical dimensions and CM voltage/current are different. Since the CM current is computed using the expressions in [12], the CM RE can be estimated using the equivalent asymmetrical dipole antenna.

The CM RE from the dipole antenna is then estimated using line integration of the CM current along the dipole arms as given in [11]

$$
E_{\theta}=\frac{\eta \beta}{4 \pi} \sin \theta \times\left\{\int_{0}^{l_{1}} \frac{I_{1}(x) e^{-j \beta r_{1}}}{r_{1}^{2}} d x+\int_{-l_{2}}^{0} \frac{I_{2}(x) e^{-j \beta r}}{r^{2}} d x\right\}
$$

where $r_{1}=\sqrt{\left(r^{2}+x^{2}-2 r x \cos \theta_{\max }\right)}$

In fact, the maximum CM RE corresponding to each frequency is obtained at certain angle, $\theta_{\max }$ which can be computed using iterative calculation for all the values of $\theta$ at the range from 0 to 180 degrees. To reduce the computational time for calculating the maximum CM RE, the angle at which the maximum CM RE is computed have to be identified first before calculating the CM RE. For that purpose, a simple analytical expression is given in [12]

$$
f(\theta)=\sin \theta\left(\int_{0}^{L_{1}} \frac{\sin \beta\left(L_{1}-|x| e^{-j k r_{1}}\right.}{r_{1}^{2}} d x+\int_{-L_{2}}^{0} \frac{\sin \beta\left(L_{2}-|x| e^{-j k r}\right.}{r^{2}} d x\right)
$$

Once the function $f(\theta)$ is computed for the certain frequency under evaluation within the range of theta angles from $0^{0}$ to $180^{\circ}$, the maximum RE can be estimated by substituting the angle theta with that selected angle with maximum value of the function $f(\theta)$. Thus, the angle $\theta_{\max }$ is the angle with maximum computed function $f(\theta)$ and it can be determined as 


$$
f\left(\theta_{\max }\right)=\max _{0 \leq \theta \leq 180} f(\theta)
$$

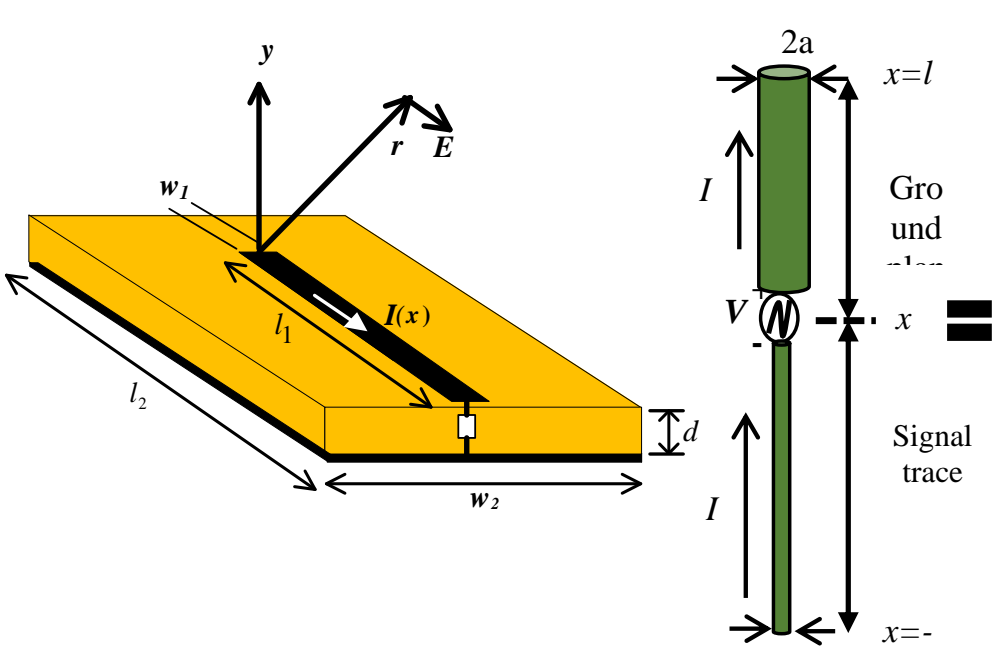

$2 b$

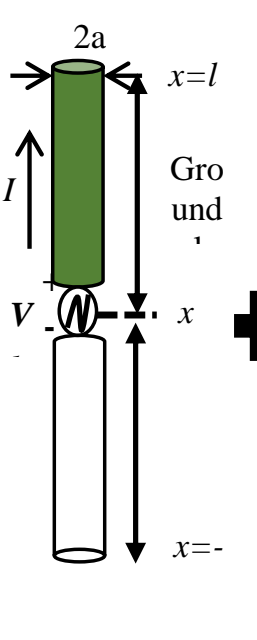

(b)

Figure 3. (a) Simple microstrip PCB for maximum CM-RE formulation (b) equivalent asymmetrical dipole antenna using imbalance difference model

\section{RESULTS AND ANALYSIS}

In order to verify the proposed methods for estimating RE from PCB traces, a simple microtrsip with a $50 \mathrm{~mm} \times 200 \mathrm{~mm}$ was fabricated. It consists of a signal trace of $1-\mathrm{mm}$ width and $150 \mathrm{~mm}$ length at the centre of the board. The return path is a ground plane on the opposite side of the PCB. Both the signal trace and ground plane are etched on a $1.6 \mathrm{~mm}$ thickness FR4 dielectric material. The signal trace is fed by $5 \mathrm{~V}-66-$ $\mathrm{MHz}$ trapezoidal signal oscillator to produce several harmonics beyond the operating frequency. A 7805 regulator was used to provide consistent $5 \mathrm{~V}$ dc to the $66 \mathrm{MHz}$ oscillator from $9 \mathrm{~V}$ battery as shown in Figure 4. To be more practical, the far-end of the trace was loaded with 100-ohm resistance.

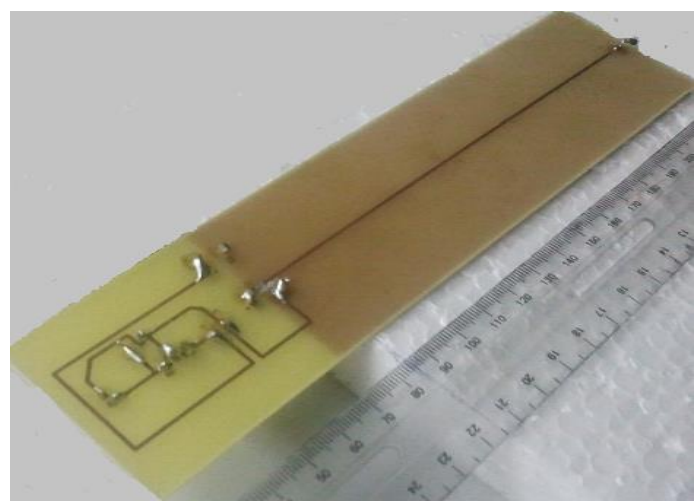

(a)

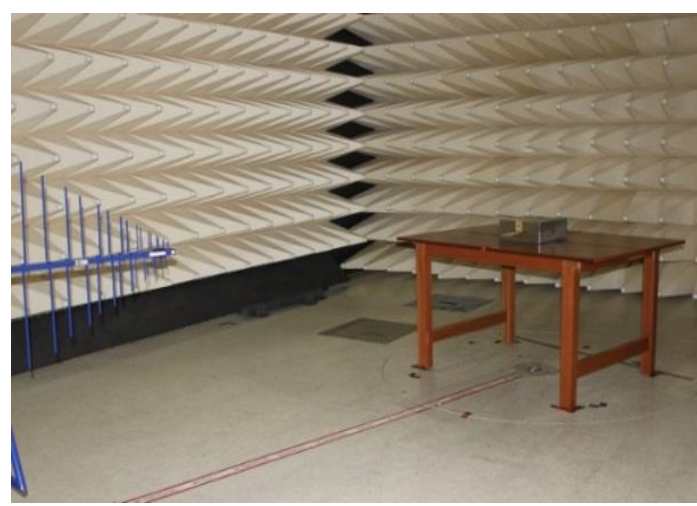

(b)

Figure 4. (a) microstrip PCB under test, (b) Measurement set-up inside SAC

The DM REs of this microstrip PCB were estimated using TRM method and Equation (4). The results of DM RE using these two methods are then verified by comparing them to that results obtained of both the conventional Paul's expression (1) and the analytical solution proposed by Leone [5] as shown in 
Figure 5(a). Very good agreements were obtained between the proposed methods and the Leone's analytical solution in [5] confirming the correctness of the proposed methods. As expected, the Equation (1) has failed to estimate accurately the DM RE of the electrically long PCB-traces. Specifically, DM radiation by the proposed method remains above $40 \mathrm{~dB}$ and reaches its peaks at $60 \mathrm{~dB}$. Its distribution does not show a sharp decline at any frequency.

On the other hand, the estimated CM emissions using the proposed expression (9) differs by about $40 \mathrm{~dB}$ compared to the conventional closed form expression (8) as shown in Figure 5(b). This observation shows the limitation of the conventional Paul's expression since it is derived based on the theory of the electrically short traces.

For further verification, the results of the proposed method are compared with the results of the existing methods. It is observed in Fig 6 that the measured RE do not exceed the upper bounds over most of the frequency range, which indicates that the proposed models are reliable for estimating the RE of PCB traces as shown in Figure 6. Moreover, they show better agreement with real measurement than do predefined models computed from Equation (1) and Equation (8) without segmentation. A good agreement between the prediction and measurement was obtained except for the higher frequencies. Such imperfection is ascribed to the fact that prediction models developed based on Equation (1) and Equation (2) are designated for electrically short traces (low frequency range).

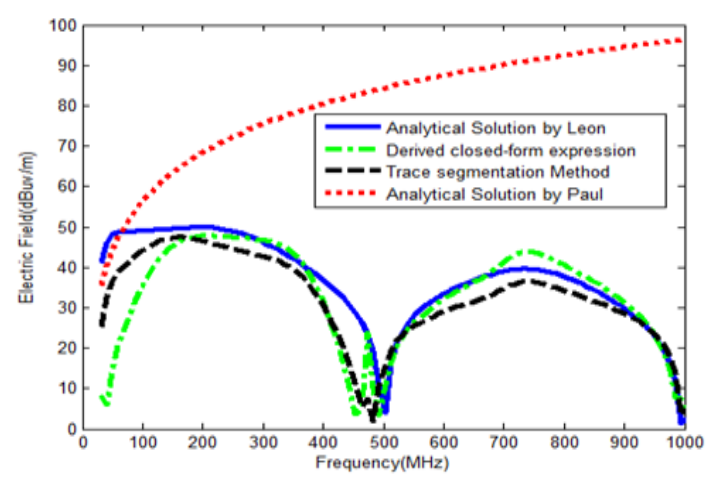

(a)

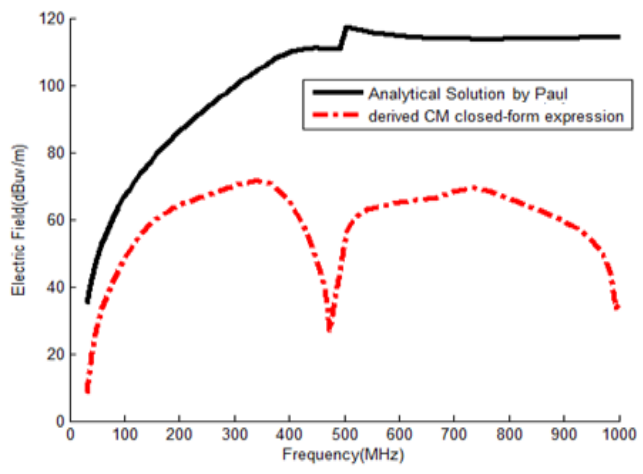

(b)

Figure 5. (a) DM RE, (b) CM RE of microstrip PCB under test using various methods

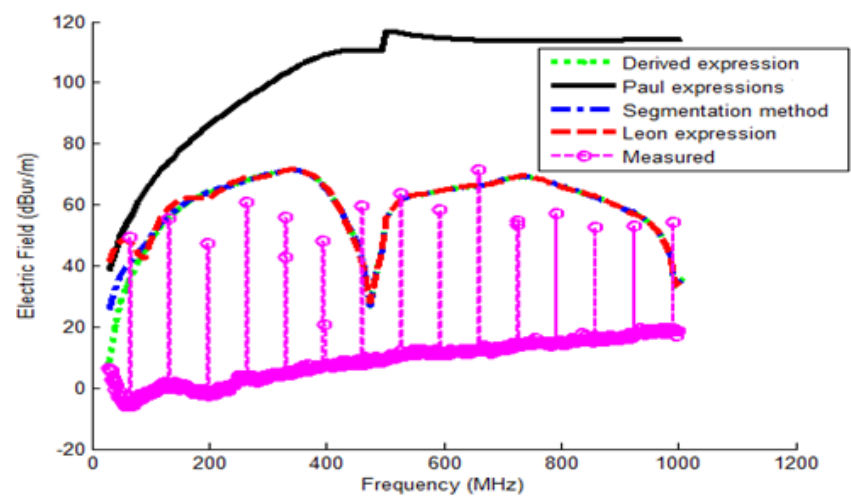

Figure 6. The total measured and estimated REs of microstrip PCB under test

Although the proposed TSM and the other previously described methods provide a good estimate of $\mathrm{RE}$, it is applicable to traces with maximum length of one wavelength. Therfore, a novel analytical solution has been developed for estimating DM RE from electrically long traces of multiple wavelengths. For validating the developed analytical solution (5), a simple microstrip PCB with one meter trace length and $2 \mathrm{~mm}$ trace width has been fabricated This trace is etched over $1.6 \mathrm{~mm}$ of FR4 dielectric material. The near end of the trace is fed through SMT-3 signal generator via shielded coaxial cable. The coaxial cable is also 
clamped with many ferrite beats around the feeding cable to prevent current to flow through the feeding cable. The far-end of the trace is terminated with $820 \mathrm{Ohm}$ since the practical design is mostly loaded with impedance. The produced feeding signal has $1 \mathrm{~V}$ amplitude within the frequency range $500 \mathrm{MHz}-3 \mathrm{GHz}$. The PCB is then placed on 1 meter $\times 1$ meter aluminum sheet to consider only the DM RE. This aluminum sheet represents the ground plane of the PCB to reduce CM RE as shown in Figure 7.

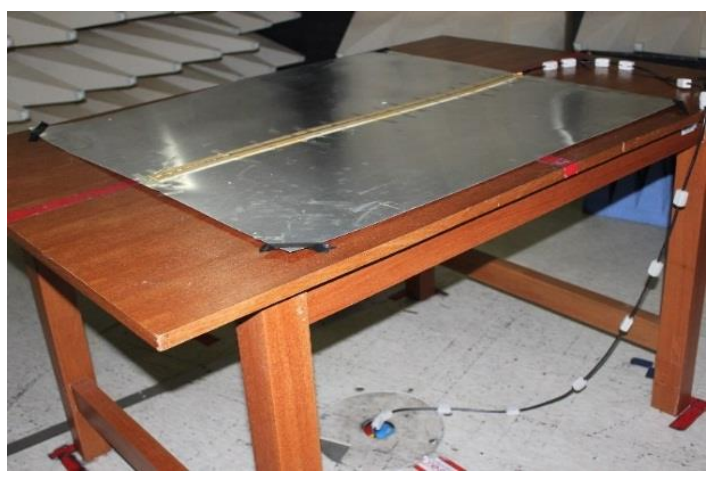

(a)

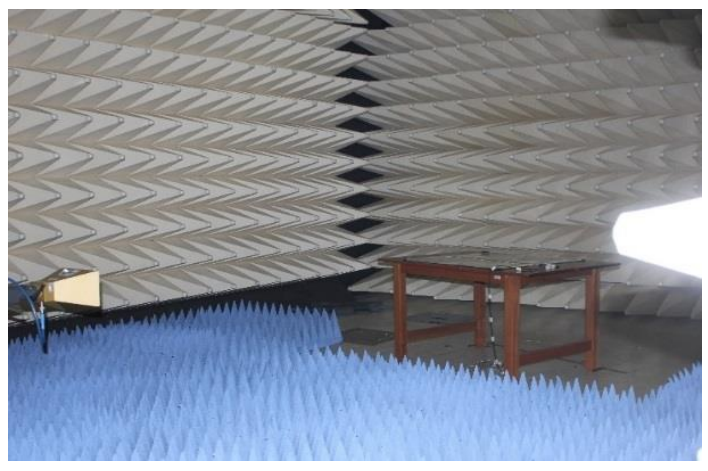

(b)

Figure 7. Measurement setup of electrically long PCB inside SAC

The REs of this PCB are then measured in SAC located at the Research Center for Applied Electromagnetics (EMcenter), Universiti Tun Hussein Onn Malaysia (UTHM). As shown in Figure 7(a), the PCB under test is placed on a $0.8 \mathrm{~m}$ tall wooden table above the ground plane in SAC and rotated $360^{\circ}$ to detect the maximum RE. A horn antenna is employed to measure the RE in the frequency range from $1 \mathrm{GHz}$ to $3 \mathrm{GHz}$ as shown in Figure 7(b). The frequency range is selected to satisfy the condition of electrically long traces where the PCB-trace becomes multiples of wave length. The distance between the PCB under test and the receiving antenna was about $3 \mathrm{~m}$ while the electromagnetic interference receiver was located outside the chamber to record the maximum $\mathrm{RE}$ as the table rotates $360^{\circ}$. The REs for frequencies below than $1 \mathrm{GHz}$ are obtained using the same measurement-setup except that the receiving antenna is replaced by a log-periodic antenna instead of horn antenna.

The estimated results are in good agreement with the measurement results as shown in Figure 8 . The proposed model successfully estimated the RE from PCB with load. There are a few decibels in discrepancy between the proposed model and the measurement. However, it still acceptable from the design point of view since it does not exceed $3 \mathrm{~dB}$ at the worst case.

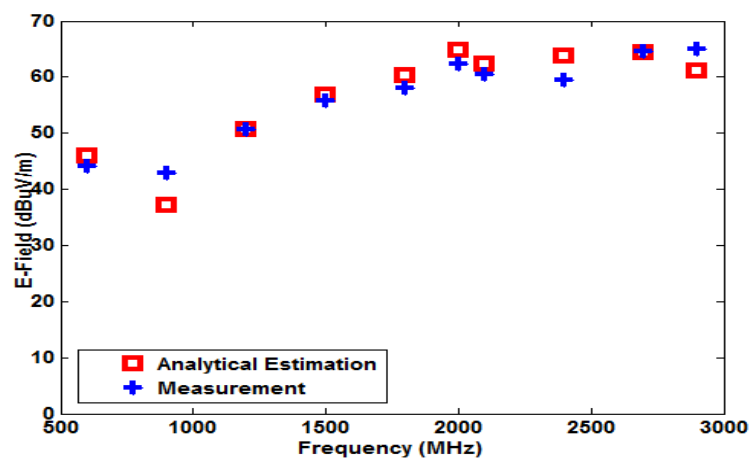

Figure 8. The measured and estimated REs of electrically long PCB-trace

\section{CONCLUSION}

In this paper, novel analytical models are presented for estimating the radiated emissions of printed circuit board traces. These analytical models are developed, for both differential model and common mode 
RE, based on dipole antenna and imbalance difference concept. Various PCBs are employed to validate these proposed models and good agreements are obtained between the results of the proposed model and the simulated/measured results. These analytical models are helful for circuit designers to avoid delay in marketing and reduce the unit cost. Thus, these models can be enhanced in future to include the other possible sources of RE. Finally, all the models are programmed to be as a software tool which can be used even for non-expert EMC users.

\section{ACKNOWLEDGEMENTS}

The authors would like to acknowledge Universiti Tun Hussein Onn Malaysia (UTHM) for their funding of this research under Postdoctoral Fellowship Contract Grant and also the RAGS research grant R069. We also would like to thank the Research Center for Applied Electromagnetics, (EMCenter), UTHM for performing the measurement of RE in the SAC.

\section{REFERENCES}

[1] Ott H. W., "Electromagnetic Compatibility Engineering”, New Jersey, USA: John Wiley \& Sons, Ltd; 2009.

[2] Paul, C. R., "Introduction to Electromagnetic Compatibility", New York, USA: Wiley; 2006.

[3] A. M. Sayegh and M. Z. Mohd Jenu, "Prediction of radiated emissions from high-speed printed circuit board traces using dipole antenna and imbalance difference model," IET Sci. Meas. Technol., vol. 10, pp. 28-37, 2016.

[4] B. W.-J. Wong and A. Cantoni, "Modeling and analysis of radiated emissions and signal integrity of capacitively loaded printed circuit board interconnections," IEEE Trans. Electronmagn. Compat., vol. 54, pp. 1087-1096, 2012.

[5] M. Leone, "Closed-form expressions for the electromagnetic radiation of microstrip signal traces," IEEE Trans. Electronmagn. Compat., vol. 49, pp. 322-328, 2007.

[6] A. M. Sayegh, M. Z. Mohd Jenu, S. Z. Sapuan and S. H. Dahlan, "Analytical solution for maximum differential-mode radiated emissions of microstrip trace," IEEE Trans. Electromagn. Compat., vol. 58, pp. 1417-1424, 2016.

[7] Balanis C. A., “Antenna Theory: Analysis and Design. Hoboken”, New Jersey, USA: John Wiley \& Sons; 2012.

[8] A. M. Sayegh and M. Z. Jenu, "Closed-form expressions for estimating maximum radiated emissions from the traces of a Printed Circuit Board," in Asia-Pacific Symposium on Electromagnetic Compatibility (APEMC)-2015, APEMC, 2015, pp. 620-623.

[9] T. Watanabe, O. Wada, T. Miyashita and R. Koga, "Common mode current generation caused by difference of unbalance of transmission lines on a printed circuit board with narrow ground pattern," IEICE Trans. Commun., vol. E83-B(3), pp. 593-599, 2000.

[10] H. W. Shim and T. H. Hubing, "Derivation of a closed-form approximate expression for the selfcapacitance of a printed circuit board trace," IEEE Trans. Electromagn. Compat., vol. 47, pp. 10041008, 2005.

[11] R. King, “Asymmetrically driven antennas and sleeve dipole,” Proceedings of the IRE Journal,vol. 38(, 1154-1164, 1950.

[12] N. Zhang, J. Kim, S. Ryu, and W. Nah, "Prediction of common-mode radiated emission of PCB with an attached cable using imbalance difference model," IEICE Transaction on Communication, vol. E98-B, pp. 638-645, 2015.

\section{BIOGRAPHIES OF AUTHORS}

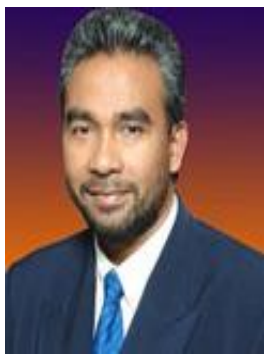

Mohd Zarar Bin Mohd Jenu received the Bachelor's degree in Engineering (electrical) from Universiti Teknologi Malaysia, Bahru, Malaysia, in 1984, and the Master's degree in Engineering and the Ph.D. degree from the University of Essex, Colchester, U.K., in 1986 and 1994, respectively. He is currently with the Research Center for Applied Electromagnetics, Universiti Tun Hussein Onn Malaysia (UTHM), Batu Pahat, Malaysia. His research interests include EMC, electromagnetic shielding, bioelectromagnetics, and computational electromagnetics. Dr. Jenu is an International Association for Radio, Telecommunications, and Electromagnetics certified EMC Engineer, serves the IEE technical panel on Electromagnetics, and was formerly the Vice-Chair of the MTT/AP/EMC Chapter IEEE Malaysia Section. He has published numerous conference proceedings as well as papers in local and international journals. To date, he has received 21 
medals and special awards at national and international exhibitions for his research products. The Center for Electromagnetic Compatibility, UTHM was his brainchild and is recognized as the first of its kind in Malaysia that pro- vides EMC testing services, consultancy, and technical supports to government agencies and industrial partners.

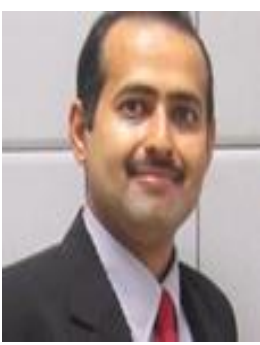

Ahmed Mohammed Sayegh received the Bachelor's(Hons.) degree in Engineering from the Sudan University of Science and Technology, Khartoum, Sudan, in 2008. He has obtained the Master's degree in Engineering and the Ph.D. degree from Universiti Tun Hussein Onn Malaysia, Batu Pahat, Malaysia, in 2012 and 2017 respectively. He has published many journal papers and conference proceedings. His current research interests include characterization of EMC radiated emissions from high-speed PCB, electromagnetic modeling, EMC of railways and computer-based modeling tools. Mr. Sayegh has received numerous medals at international exhibitions for his research products

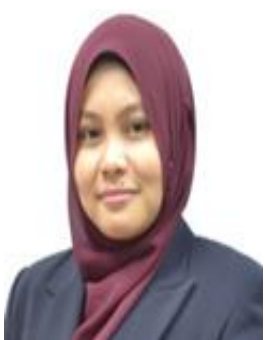

Syarfa Zahirah Sapuan received the B.Eng.(Hons.) degree in electrical engineering from Kolej Universiti Tun Hussein Onn Malaysia (UTHM), Batu Pahat, Malaysia, in 2003, the M.Sc. degree in communication engineering from Nanyang Technological University, Singapore, in 2009, and the Ph.D. degree in electrical engineering from UTHM in 2014. She is currently a Senior Lecturer at the Communication engineering Department, UTHM, where she is also a Principle Researcher of the Electromagnetic Compatibility Research Cluster at the Research Center for Applied Electromagnetics. Her research interests include electromagnetic compatibility, antenna calibration, and radio frequency measurements 